\title{
Using Recursively Defined Subsets of the Power Set of $P$ to Show an Inequality betweenP andBPP
}

\author{
Austen Bentley
}

\begin{abstract}
In this paper we resolve the question of whether or not $P$ and BPP are unequal.We do this by showing the existence of a set that is a subset of $P$ and not in $P$, then using a property of the definition of this set to show it is within BPP.
\end{abstract}

\section{Introduction}

In this paper we use the definition of BPP in terms of NP from 28 and use aproperty of this definition and proof of the existence of a certain class of setsand their non inclusion in $\mathrm{P}$, followed by their inclusion in $\mathrm{BPP}$, and the trivialproof that $\mathrm{P}$ is an improper subset of BPP to show $\mathrm{P}$ is a proper subset of BPP.

\section{Informal outline}

We assume the definition of addition, subtraction and the complexity classes, however we assume a more abstract set of numerical definition as this allows forgreater expressive capability (i.e changing base without changing context). Weassume the definition of BPP in terms of NP seer28s. Our first theorem is that Pis an improper subset of BPP, this proof is trivial as if $\mathrm{a}$ is an element of $\mathrm{P}$ thenthere exists a polynomial time deterministic turing machine that solves a. Whereobviously if there exists a polynomial time deterministic turing machine thatsolves a then there exists a polynomial time non deterministic turing machinesuch that if $b$ is in a then at least two thirds of the computation paths areaccepting and if $b$ is not in a then less that one third of the computation pathsaccept. Our next theorem is that there exists a subset of P defined using the setof elements in a (where a is solvable by the polynomial time deterministic turingmachine b) such that every polynomial non deterministic accepting computationpaths in $\mathrm{c}$ is in $\mathrm{d}$ where $\mathrm{a}$ is a subset of $\mathrm{d}$ and $\mathrm{d}$ is in not within $\mathrm{P}$ and the stateset input alphabet et al of $\mathrm{c}$ is the same as that for $\mathrm{b}$. The proof of this is simpleby constructing the functions $\mathrm{f} 1$ and $\mathrm{f} 2$ see section 6. Theorem 3 is the existenceof a subset of P that is within BPP but not P. We prove this from the fact thatif the set we proved with theorem 2 is not exists then it is not within P thenit is within BPP and we have obviously previously shown its existence. Our final theorem and its proof are trivial, that is $\mathrm{P}$ is a proper subset of BPP, wehave from theorem one that $\mathrm{P}$ is an improper subset of BPP and we have fromtheorem three that there exists a set in BPP that is not in P.

\section{Informal definitions and axioms}

$$
\text { Definition.1. }
$$

$(a \oplus b)$ iff $(T(a) \neq T(b))$ where obviously $\mathrm{T}$ is the binary truth function of the language

$$
\text { Definition.2. }
$$

$a \wedge b$ iff $((T(a)=T(b))(T(a)=1))$ where $T$ is again the binary truth function of the language

$$
\text { Definition.3. }
$$

$a \vee b$ iff ( ( $\mathrm{a}$ and $\mathrm{b}) \oplus(a \oplus b))$ Where in this section a and $\mathrm{b}$ is equivalent to $a \wedge b$

$$
\text { Definition.4. }
$$

$a=\{b \mid c[d \mid e]\}$ iff $\mathrm{f}$ is in a iff $\mathrm{d}$ replaced by $\mathrm{f}$ in the statement $\mathrm{c}$ is true

$$
\text { Definition.5. }
$$

set[a] iff there exists $c$ and $d$ such that $(a=\{b \mid c[d \mid b]\})))$

$$
\text { Definition.6. }
$$

string $[a, b]$ iff $b$ is a set such that for all $c$ and for all $d$ such that $c$ and $d$ are in $\mathrm{b}(c d \oplus d c)$

$$
\text { Definition.7. }
$$

$\operatorname{ath}(\mathrm{b}, \mathrm{c})=\mathrm{d}$ iff $a<|c|$ where $\mathrm{a}$ is in $\mathbb{N}$ and $a=|\{e \mid(e \in c(e b d))\}|$ 


\section{Definition.8.}

$\mathrm{a}=\mathrm{b}$ iff for all $\mathrm{c}, \mathrm{d}$ such that string[c,d] and $\mathrm{a}$ is in $\mathrm{d}$ where $\mathrm{c}, \mathrm{d}$ is true $\mathrm{c}, \mathrm{d}[\mathrm{a}-\mathrm{b}]$ is true for all e,f such that string[e,f] such that $b$ is in $f$ and $e, f$ is true e,f[b-a] is true

$$
\text { Definition.9. }
$$

$a[b \mid c]=d$ iff a is a string and $\mathrm{d}$ is a string and there exists e such that the eth element of $\mathrm{a}$ is equal to $\mathrm{b}$ and the eth of $\mathrm{d}$ is equal to $\mathrm{c}$ such that for all $\mathrm{f}$ where $f \neq e$ the $\mathrm{fth}$ element of a is equal to the $\mathrm{fth}$ element of $\mathrm{d}$

\section{Definition.10.}

$\mathrm{a} / \mathrm{b}=\mathrm{c}$ iff for all $\mathrm{d}$ where $\mathrm{d}$ is in a and $\mathrm{d}$ is not in $\mathrm{b}$ we have $\mathrm{d}$ in $\mathrm{c}$

\section{Definition 11.}

class[a] iff there exists $b$ such that $b$ is in a such that $b$ is a set

\section{Definition.12.}

$a \subseteq b$ iff if $\mathrm{c}$ is in a then $\mathrm{c}$ is in $\mathrm{b}$ and $\mathrm{b}$ is a set or class(where in this section a or $\mathrm{b}$ is equivalent to $a \vee b$ and a or mutually exlusive or os equivalent to $a \oplus b$

\section{Definition.13.}

$a \subset b$ iff if a is not equal to $\mathrm{b}$ and $\mathrm{c}$ is in a then $\mathrm{c}$ is in $\mathrm{b}$

\section{Definition.14.}

$a \times b=c$ iff $\mathrm{d}$ is in $\mathrm{c}$ iff $\mathrm{d}=\mathrm{e}, \mathrm{f}$ and $\mathrm{e}$ is in a such that $\mathrm{f}$ is in $\mathrm{b}$

\section{Definition.15.}

$S_{b}^{a}(c)=d$ iff a,b is a string where $\mathrm{c}$ is in $\mathrm{b}$ and $\mathrm{d}$ is in $\mathrm{b}$ such that cad and there doesn't exists f such that fad and caf

$$
\text { Definition.16. }
$$

$a \sim^{b, c} \mathrm{~d}$ iff for all c,d such that c,d is a string and $\mathrm{a}$ is in $\mathrm{d}$ where $\mathrm{c}, \mathrm{d}$ is true it is not true that if $c, d$ is true then gbh where $g$ is in $c$ and $h$ is in $c$ however it is true that where $c, d[a-b]$ is true such that for all e, f where e, $f$ is a string and $b$ is in $f$ and e,f is true it is not true that if e,f is true the where ibj and $i$ is in $f$ and $\mathrm{j}$ is in $\mathrm{f}$ it is true that $e, f[b \mid a]$

\section{Definition.17.}

$a=\{b, c\}(\mid \notin c($ string $[b, c])))$ iff $\mathrm{d}$ is in a iff $(\mathrm{d}=\inf (\mathrm{b}, \mathrm{c})$ or $\mathrm{d}=\mathrm{sup}(\mathrm{b}, \mathrm{c}))$ or there exists e such that $e \simeq^{b, c}=$, and there exists f such that $f \simeq^{b, c}=$, where $\left(S_{c}^{b}(e)=d\right.$ and $S_{c}^{b}(d)=f$

$$
\text { Definition. } 18 \text {. }
$$

$\mathrm{b}, \mathrm{c}$ is a string where $\mathrm{a}$ is in $\mathrm{c}$ and $\mathrm{a}=\mathrm{b}, \mathrm{c}$ iff $(\mathrm{c}=\{\mathrm{a}\})$

$$
\text { Definition.19. }
$$

$\mathrm{acb}=\mathrm{c}, \mathrm{d}$ where $\mathrm{c}, \mathrm{d}$ is a string iff $\mathrm{b}$ is in $\mathrm{d}$ and $\mathrm{a}=\mathrm{c}, \mathrm{e}$ where $\mathrm{c}, \mathrm{e}$ is a string and $\mathrm{e}=\mathrm{d} /\{\mathrm{b}\}$ such that for all $\mathrm{f}$ such that $\mathrm{f}$ is in e we have feb

$$
\begin{aligned}
& \text { deci }=\{0,1,2,3,4,5,6,7,8,9\} \begin{array}{l}
\text { Definition.20. } \\
\text { Definition.21. }
\end{array} \\
& <\text {, deci }=0<1<2<3<4<5<6<7<8<9
\end{aligned}
$$


Definition. 22 .

$\mathbb{N}_{a, b}=\left\{a, c \mid\left(\right.\right.$ string $\left.\left.[a, c]\left(\forall d\left(d \in c\left(d \simeq \simeq^{a, c} e(e \in b)\right)\right)\right)\right)\right\}$ iff a,b is a string

Definition.23.

$a: b \rightarrow c$ iff for all $\mathrm{d}$ where $\mathrm{d}$ is in $\mathrm{b}$ there exists e such that $\mathrm{e}$ is in $\mathrm{c}$ and $\mathrm{a}(\mathrm{d})$ is equal to e and for all $\mathrm{f}$ such that $\mathrm{f}$ is not in $\mathrm{b}$ it is true that for all $\mathrm{g}$ we have $\mathrm{a}(\mathrm{f})$ is not equal to $\mathrm{g}$

$$
\text { Definition. } 24 \text {. }
$$

injection $[a, b, c]$ iff $a: b \rightarrow c$ and if $\mathrm{a}(\mathrm{d})=\mathrm{a}(\mathrm{e})$ then $\mathrm{d}$ is equal to $\mathrm{e}$

$$
\text { Definition. } 25 .
$$

surjection $[a, b, c]$ iff $a: b \rightarrow c$ where for all $\mathrm{d}$ such that $\mathrm{d}$ is in $\mathrm{c}$ there exists $\mathrm{e}$ where $e$ is in $b$ such that $a(e)=d)$

$$
\text { Definition. } 26 .
$$

bijection $[a, b, c]$ iff injection $[a, b, c]$ and surjection $[a, b, c]$

Definition.27.

$|a|<|b|$ iff there exists $c$ such that injection $[c, a, b]$

\section{Definition.28.}

$|a|=|b|$ iff there exists c such that bijection $[c, a, b]$

\section{Definition.29.}

$a, b<\mathbb{N}_{a, c} a, d$ iff a,b is in $\mathbb{N}_{a, c}$ and a,d is in $\mathbb{N}_{c, d}$ and a,b is a string such that a,d is a string where $((|b|<|d|) \oplus|b|=|d|$ such that there exists e such that for all $\mathrm{f}$ where $f<e$ and $\mathrm{fth}(\mathrm{a}, \mathrm{b})=\mathrm{fth}(\mathrm{a}, \mathrm{d})$ we have eth(a,b) $<\mathbb{N}_{a, c}$ eth(a,d)

\section{Definition. 30}

$\mathbb{N}_{a, b}(c)=d$ iff $c$ is in $\mathbb{N}_{a, b}$ and $\left|\left\{e \mid\left(e<\mathbb{N}_{a, b} c\right)\right\}\right|=d$

Definition.31.

$\mathbb{N}=\left\{a \mid\left(\exists b\left(\exists c\left(\right.\right.\right.\right.$ string $\left.\left.\left.\left.[b]\left(\mathbb{N}_{b}(c)=a\right)\right)\right)\right)\right\}$

Definition.32.

$T M[a, b, c, d, e, f, g]$ iff $\mathrm{c}$ is in $\mathrm{b}$ and $d=b / c$ such that $g \subseteq a$ where $\mathrm{f}$ is in a and $e:(a / g) \times b \times\{T M L, T M R\} \rightarrow a \times b \times\{T M L, T M R\}$ and $|g|=\mathbb{N}_{<, \text {deci }}(2)$

\section{Definition.33.}

$a \lessgtr_{b, c} d$ iff a is a set or a class and $\mathrm{d}$ is a set or a class and for all e such that $\mathrm{e}$ is in a and $e \simeq \simeq^{b, c} f$ it is true that $\mathrm{f}$ is in $\mathrm{d}$

\section{Definition.34.}

compfunc $[a, b]$ iff, $\mathrm{a}$ is a string and for all $\mathrm{c}$ such that $\mathrm{b}(\mathrm{c})=\mathrm{d}(\mathrm{e}(\mathrm{c}))$ and compfunc [f,e] where $\mathrm{d}=\sup (\mathrm{a})$ we have $f=a /\{d\}$

$$
\text { Definition.35. }
$$

$\operatorname{DTM} M[a, b, c, d, e, f, g, h, i, j]$ iff $, \mathrm{j}=\mathrm{A}, \mathrm{B}, \mathrm{C}$ and $\mathrm{A}$ is in $\mathrm{g}$ where $\mathrm{B}$ is in $\mathrm{b}$ and $\mathrm{C}$ is in $\mathbb{I N}$ such that $T M[a, b, c, d, e, f, g]$ and , i is a string and , $\mathrm{j}$ is a string where $i \lesssim b$ and compfunc $[h, k]$ where $k\left(f,, i, \mathbb{N}_{<, \text {deci }}(0)\right)=, j$ and $|h|$ is in $\mathbb{I N}$ and $\left(\operatorname{lth}(, \mathrm{h})(\mathrm{m}, \mathrm{n}, \mathrm{o})=\mathrm{p}, \mathrm{q}, \mathrm{r}\right.$ iff $\mathrm{o}=\mathrm{IN}_{<, \text {deci }}(\mathrm{O})$ and $\mathrm{e}(\mathrm{m}, \inf (\mathrm{n}))=\mathrm{p}, \mathrm{IN}_{<, \text {deci }}(\mathbf{1}), \mathrm{TML}$ where $\mathrm{r}=\mathbb{N}_{<, \text {deci }}(\mathrm{O})$ and $\inf (\mathrm{q})=\mathrm{c}$ such that for all $\mathrm{s}$ such that $r<\mathrm{N} s$ where $\operatorname{sth}(n)=S_{\mathrm{IN}}^{<}(s) t h(q) \oplus \operatorname{oth}(\mathrm{n})=\sup (\mathrm{n})$ and $\mathrm{e}(\mathrm{m}, \sup (\mathrm{n}))=\mathrm{p}, \operatorname{sth}(\mathrm{q}), \mathrm{TMR}$ where $S_{\mathbb{N}}^{<}(0)=r$ and $\sup (\mathrm{q})=\operatorname{rth}(\mathrm{q})$ and $\operatorname{rth}(\mathrm{q})=\mathrm{c}$ such that for all $\mathrm{t}$ such that $\mathrm{t}<_{\mathbb{N}} \mathrm{O}$ and $\operatorname{tth}(\mathrm{n})=\operatorname{tth}(\mathrm{q}) \oplus o \neq \mathbb{N}_{<, \text {deci }}(0)$ and $\mathrm{e}(\mathrm{m}, \mathrm{oth}(\mathrm{n}))=\mathrm{p}, \mathrm{oth}(\mathrm{q}), \mathrm{TML}$ such that for all s such that $s \neq o$ it is true that $\operatorname{sth}(\mathrm{n})=\operatorname{sth}(\mathrm{q}) \oplus \operatorname{oth}(n) \neq \sup (n)$ and $(\mathrm{e}(\mathrm{m}, \operatorname{oth}(\mathrm{n}))=\mathrm{p}$,oth $(\mathrm{q}), \mathrm{TMR}$ such that for all s such that $s \neq o$ it is true that $\operatorname{sth}(n)=\operatorname{sth}(q)$ 


\section{Definition.36.}

$N D T M[a, b, c, d, e, f, g, h, i, j]$ iff for all $\mathrm{k}$ such that $\mathrm{k}$ is in $\mathrm{e}$ it is true that $T M[a, b, c, d, k, f, g]$ and compfunc $[k, l]$ and $l\left(f, l, \mathbb{N}_{<, \text {deci }}(0)\right)=, j$ where $, \mathrm{j}=\mathrm{A}, \mathrm{B}, \mathrm{C}$ such that $A$ is in $\mathrm{g}$ and $\mathrm{B}$ is in $\mathrm{b}$ where $\mathrm{C}$ is in $\mathrm{IN}$ such that for all $\mathrm{m}$ such that $\mathrm{m}<_{\mathbb{N}}|h|$ there exists $\mathrm{n}$ such that $\mathrm{n}$ is in e and $\mathrm{mth}(\mathrm{h})(\mathrm{o}, \mathrm{p}, \mathrm{q})=\mathrm{r}, \mathrm{s}, \mathrm{t}$ such that $\left(\left(\left(\mathrm{n}(\mathrm{o}, \mathrm{qth}(\mathrm{p}))=\mathrm{r}, \mathrm{qth}(\mathrm{s}), \mathrm{u}\right.\right.\right.$ and $q \neq \mathbb{N}_{<, \text {deci }}(0)$ such that $\left(u=T M L\left(S_{\mathbb{N}}^{<}(t)=\right.\right.$ $q)) \oplus\left(q \operatorname{th}(p) \neq \sup (p)\right.$ andu $\left.\left.=\operatorname{TMR}\left(S_{\mathrm{N}}^{\mathrm{N}}(q)=t\right)\right)\right) \oplus(n(o, q \operatorname{th}(p))=r$, uth $(s), v$ and $u=\mathbb{N}_{<, \text {deci }}(1)$ such that $\inf (\mathrm{s})=\mathrm{c}$ such that for all $\mathrm{w}$ such that $w \operatorname{th}(p)=$ $S_{\mathrm{IN}}^{<}(w) \operatorname{th}(s)$ and $\mathrm{v}=\mathrm{TML}$ where $\left.\left(S_{\mathrm{IN}}^{\wedge}(t)=q\right)\right) \oplus \mathrm{u}=\mathrm{q}$ and $S_{\mathrm{IN}}^{\times}(q) \operatorname{th}(s)=\sup (s)$ such that $\mathrm{qth}(\mathrm{p})=\sup (\mathrm{p})$ and $\mathrm{v}=\mathrm{TMR}$ and $\sup (\mathrm{s})=\mathrm{c}$ such that for all $\mathrm{w}$ such that $w<\mathbb{N} q$ and $\mathrm{wth}(\mathrm{p})=\mathrm{wth}(\mathrm{s})$

\section{Definition. 37 .}

$a=b^{c}$ where $a \in \mathbb{N}$ iff compfunc[d,e] and $e\left(\mathbb{N}_{<, \text {deci }}(1)\right)=a$ such that $|d|=c$ such that for all $\mathrm{f}$ such that $f \operatorname{th}(, d)(g)=$ iv $(b, g)$

\section{Definition. 38 .}

$a(b)=O_{c}(d(b))$ iff there exists e and there exists $\mathrm{f}$ such that $\mathrm{e}$ is in $\mathrm{IN}$ and $\mathrm{f}$ is in $\mathbb{N}$ such that for all $\mathrm{g}$ such that $\mathrm{g}$ is in $\mathrm{c}$ and $a(g) \leqslant_{\mathrm{N}} \mathbb{N}\left(e, d(g)^{f}\right)$

Definition.39.

$|a| \leqslant|b|$ iff $((|a|=|b|) \oplus(|a|<|b|))$

Definition.40.

$+_{\mathbb{N}}(a, b)=c$ iff $c$ is in $\mathbb{N}$ and $\mathrm{a}$ is in $\mathbb{N}$ and $\mathrm{b}$ is in $\mathbb{N}$ and $\left\{d \mid\left(d<_{\mathbb{N}} c(a \leqslant \mathbb{N} d)\right)\right\}$

Definition. 41

iN $(a, b)=c$ iff $\mathrm{c}$ is in $\mathrm{IN}$ and $\mathrm{a}$ is in $\mathrm{N}$ and $\mathrm{b}$ is in $\mathrm{N}$ such that compfunc[d,e] where $|d|=b$ and $e\left(\mathbb{N}_{<, \text {deci }}(0)\right)=c$ such that for all $\mathrm{f}$ such that for all $\mathrm{g}$ it is true that $f \operatorname{th}(d)(g)=+\mathbb{N}_{<, \text {deci }}(a, g)$

\section{Definition.42.}

$P=\{k \mid((\operatorname{set}[k] \vee \operatorname{class}[k])(\exists a(\exists b(\exists c(\exists d(\exists e(\exists f(\exists g(\exists l(\exists m(\forall i)(\exists h(\exists j(N D T M[a, b, c, d, e, f, g, h, i, j](|h|=\mathrm{iN}$ $\left(l,|i|^{m}\right)(j=n, o, p(n=\inf (, g) \Leftrightarrow i \notin k)((n=\sup (, g) \Leftrightarrow i \in k)(n=$ $\sup (, g) \oplus n=\operatorname{in} f((, g))))())))()))())))))))\}$

\section{Definition.43.}

$N P=\left\{k \mid\left(\exists a(\exists b) \exists c\left(\exists d\left(\exists e\left(\exists f\left(\exists g\left(\exists l(\exists m)(\forall i), i \in k\left(\exists h\left(\exists j\left(N D T M[a, b, c, d, e, f, g, h, i, j](|h|)_{\mathrm{iN}}\right.\right.\right.\right.\right.\right.\right.\right.\right.\right.$ $\left(l,|i|^{m}\right)(j=n, o, p(n=\inf (, g) \Leftrightarrow i \notin k)((n=\sup (, g) \Leftrightarrow i \in k)(n=$ $\sup (, g) \oplus n=\inf ((, g)))))()))()))()))))))\}$

Defintion. 45 .

$\mathbb{Z}_{a, b}=\left\{a, c \mid\left(\operatorname{string}[a, c]\left(a, c \in \mathbb{N}_{a, b} \oplus\left(a, c=-\operatorname{ad}\left(\operatorname{string}[a, d]\left(a, d \in \mathbb{N}_{a, c}\right)\right)\right)\right)\right)\right\}$

Defintion.46.

$\mathbb{Z}_{a}(b)=c \Leftrightarrow\left(\mathbb{N}_{a}(b)=c \oplus\left(b=-\operatorname{de}\left(\operatorname{string}[d, e]\left(c=-\mathbb{N}_{a}(d, e)\right)\right)\right)\right)$

Defintion.47.

$$
\mathbb{Z}=\left\{a \mid\left(\exists b\left(\exists c\left(\mathbb{Z}_{b}(c)=a\right)\right)\right)\right\}
$$

Defintion. 48 .

$\mathbb{R}_{a, b}=\left\{a, c \mid\left(\right.\right.$ string $[a, c]\left(\left(a, c \in\right.\right.$ mathds $Z_{a, b} \oplus\left(. \in c\left(\forall e\left(e \in c\left(\sup (a, c) \neq\left(\forall g\left(g \in c\left(g \simeq^{a, c} h(g \neq .(h \in b\right.\right.\right.\right.\right.\right.\right.$

)$\left.\left.\left.)))))))) \oplus\left(\inf (a, c)=-\left(\sup (a, c) \neq .\left(\forall g\left(g \in c\left(g \neq-\left(g \neq .\left(g \simeq^{a, c} h(h \in h(h \in b))\right)\right)\right)\right)\right)\right)\right)\right)\right)\right\} \Leftrightarrow \operatorname{string}[a, b]$

Defintion.49.

$$
\mathbb{R}_{a}(b)=\mathbb{Z}_{a}(b) \Leftrightarrow b \in \mathbb{Z}_{a}
$$

Definition.50.

$$
\begin{aligned}
& {\stackrel{+}{\mathbb{Z}_{a, b}}}(a, c, a, d)=a, e \Leftrightarrow\left(\left(_{\mathbf{Z}_{a, b}}^{+}(a, c, a, d)=a, e \oplus\left(_{\mathbf{Z}_{a, b}}^{+}(a, c /\{\inf (a, c)\}, a, d /\{\inf (a, d)\})=a, e\right.\right.\right. \\
& \left.(\inf (a, c)=-(\inf (a, d)=-)))) \oplus\left(\inf (a, d)=-\left(\inf (a, c) \neq-\left(-\mathbb{N}_{a, b}(a, c, a, d /\{\inf (a, d)\})\right)\right)\right)\right) \\
& \text { Defintion.51. } \\
& \mathbb{R}=\left\{a \mid\left(\exists d\left(\exists b, c\left(\text { string }[b, c]\left(\mathbb{R}_{b, c}(d)=a\right)\right)\right)\right)\right\}
\end{aligned}
$$


Definition. 52 .

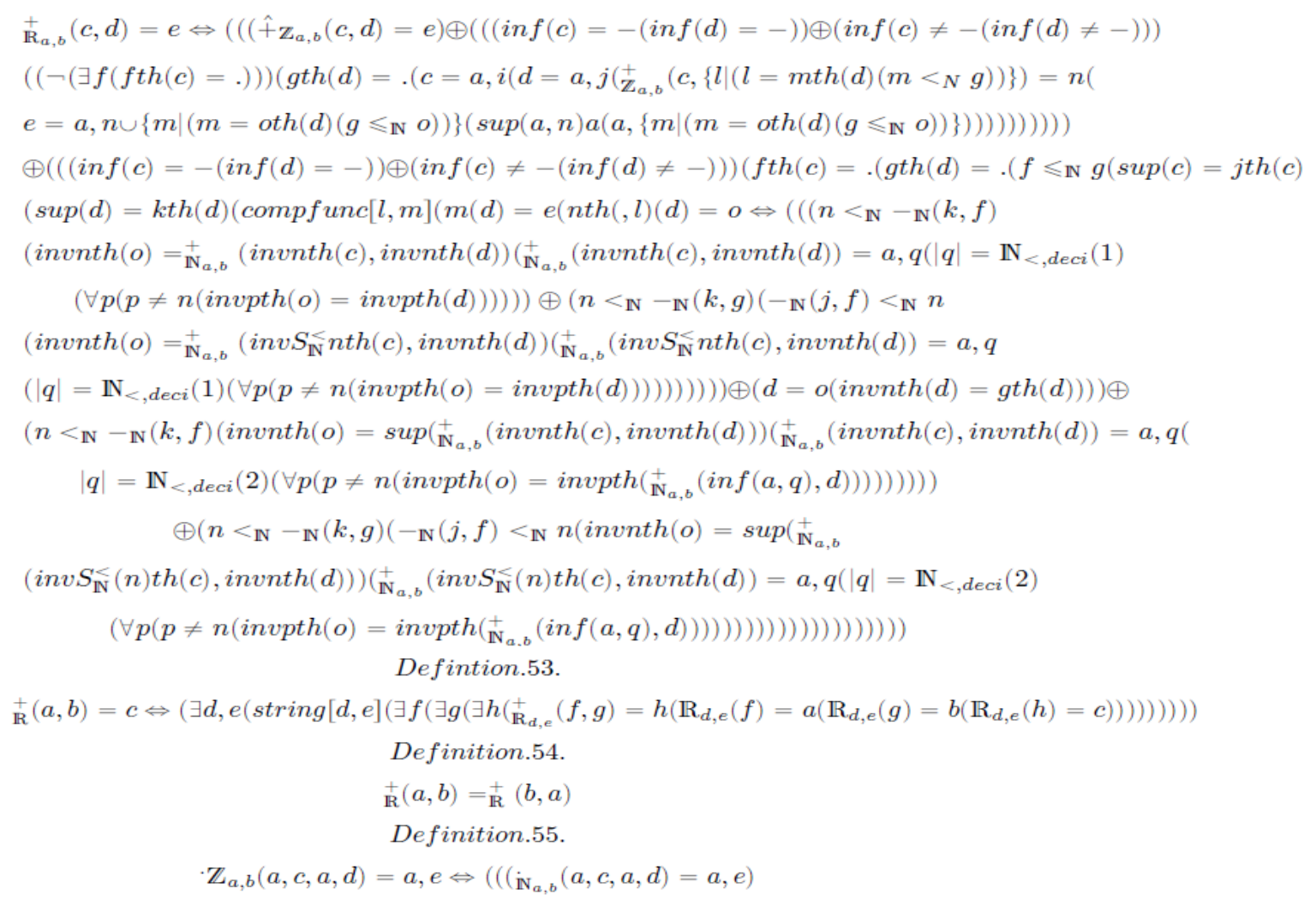




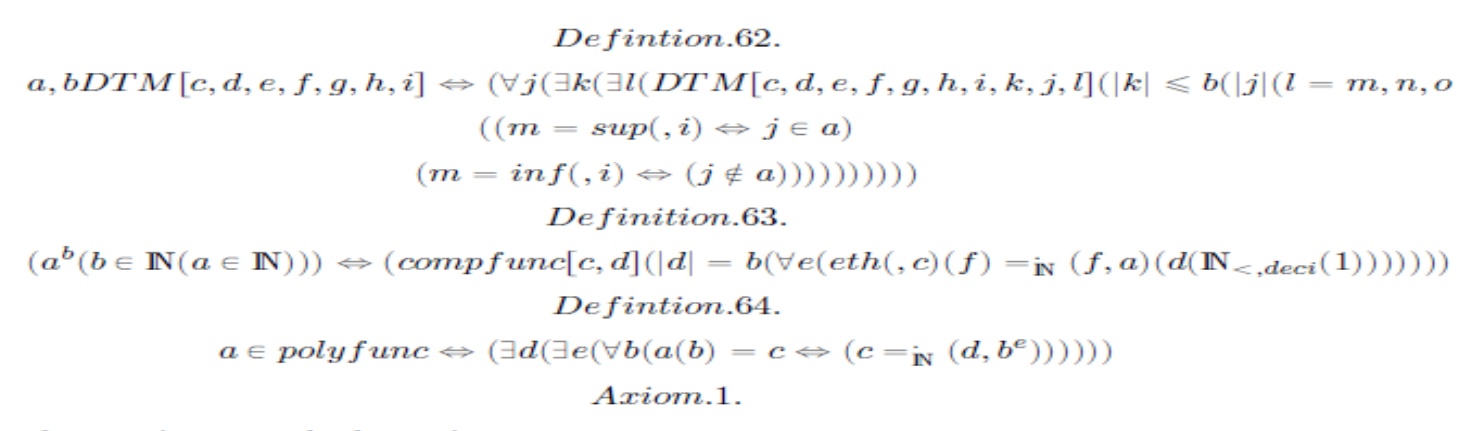

there exists a such that $a$ is a set

Axiom.2.

there exists a such that a is a class

$$
\begin{aligned}
& P \neq \varnothing \quad \text { Axiom.3. } \\
& \text { Axiom.4. } \\
& \text { there exists a such that } a=\mathbb{N}<\text { deci } \\
& \text { Axiom.5. } \\
& \text { there exists a such that } a=\mathbb{N}
\end{aligned}
$$

\section{Informal Theorems and proof} Th.1. $\subseteq B P P$

\footnotetext{
$\operatorname{Pr} .1 .((a \in P) \Rightarrow(\exists b, c, d, e, f, g, h(a, j D T M[b, c, d, e, f, g, h](j \in$ polyfunc $))))$

$\wedge(((\exists b, c, d, e, f, g, h(a, j D T M[b, c, d, e, f, g, h](j \in$ polyfunc $))) \Rightarrow(\exists x(\exists y(x \in \mathbb{R}(y \in \mathbb{R}(\exists b, c, d, e, f, g, h,(\forall i($ $j(i)=\left|\left\{l \mid\left(\exists m\left(N D T M[b, c, d, e, f, g, h, l, k, m]\left(|l| \leqslant_{\mathbf{R}} \mathbf{R}\left(x,|k|^{y}\right)\right)\right)\right)\right\}\right|$

$\left(\left((k \in a) \Rightarrow\left(\dot{\mathrm{R}}\left(j(k), \mathbb{R}_{<, \text {deci }}\left(/_{\mathbf{R}, \text { deci }}(2,3)\right) \leqslant \mathbf{R} \mid\{l \mid(\exists m(N D T M[b, c, d, e, f, g, h, l, k, m]\right.\right.\right.\right.$

$\left.\left.\left.\left.\left.\left.\left(m=n, o, p\left(n=\sup (, h)\left(|l| \leqslant_{\mathrm{R}} \mathbf{R}\left(x,|k|^{y}\right)\right)\right)\right)\right)\right)\right\} \mid\right)\right)\right)\left(\left(k \notin a\left(k \in c^{*}\right)\right) \Rightarrow(\mid\{l \mid(\exists m(N D T M[b, c, d, e, f, g, h, l, k, m]\right.$

$\left.\left.\left.\left.\left.\left.\left.\left.\left.\left.\left.\left.\left.\left.\left(m=n, o, p\left(n=\sup (, h)\left(|l| \leqslant_{\mathbf{R}} \mathbf{R}\left(x,|k|^{l}\right)\right)\right)\right)\right)\right)\right\} \leqslant_{\mathbf{R} R}\left(\mathbb{R}_{<, \text {deci }}\left(/ \mathbb{R}_{<, \text {deci }}(1,3)\right), j(k)\right)\right)\right)\right)\right)\right)\right)\right)\right)\right)\right)\right\}$

Th.2. $(\exists a(a \subseteq P(\operatorname{string}[, a](\operatorname{string}[, b](|a|=|b|(\forall j(\forall k(\exists l(D T M[c, d, e, f, g, h, i, j \operatorname{th}(, b), k, l]$

$(l=m, n, o((m=\sup (, i) \Leftrightarrow(k \in j \operatorname{th}(, a)))((m=\inf (, i) \Leftrightarrow(k \notin j \operatorname{th}(, a)))(\forall j(N D T M[c, d, e, f, b, h, i, j, k, l]$

$\left.\left(|j| \leqslant_{\mathbf{R} R}\left(\mathbb{R}_{<, \text {deci }}(10),|k|^{\mathbf{R}_{<, d e c i}(100)}\right)\right)\right)(\{k \mid(k \in a \vee(N D T M[c, d, e, f, b, h, i, j, k, l]$

$(l=m, n, o(m=\sup (, i)))))))\} \notin P())))))))))))$

$\operatorname{Pr.2.}\left(\left(\left(\left((a(b, c)=d \Leftrightarrow b \operatorname{th}(, c)=d)\left(a_{1}=\{a(z, b) \mid(z \in \mathbb{N}(b \in P(b, d D T M[e, f, g, h, y \operatorname{th}(, i), j, k]\right.\right.\right.\right.\right.$

$(d \in \operatorname{polyfunc}(\forall l(l \in a) \exists m(m \in \operatorname{polyfunc}(\exists n(n \in q(l, m D T M[e, f, g, h, n, j, k](\forall o(\forall p(p \in$ polyfunc (

$\left.\left.\left.o, p N D T M[e, f, g, h, q, j, k](\forall r(r \in o(r \in a))))))))))))))))))\}\left(\{01\} \in a_{1}\left(a_{1} \in P\right)\right)\right)\right) \Rightarrow(a \in a)\right) \wedge$

$\left(\left(f_{1}(d, 0)=0,0, T M R\left(f_{1}(0,1)=\sup (, h), 1, T M R\left(f_{2}(d, 1)=0,0,, T M R\left(f_{2}(0,0)=\sup (, h), 0, T M R\right.\right.\right.\right.\right.$

$\left(f_{1}(d, 1)=2,0, T M R\left(f_{1}(2,0)=\inf (, h), 0, T M R\left(f_{1}(2,1)=\inf (, h), 0, T M R\left(f_{2}(0,1)=\inf (, h), 0, T M R(\right.\right.\right.\right.$

$f_{2}(2,0)=\inf (, h), 0, T M R\left(f_{2}(2,1)=,\inf (, h), 0, T M R\left(f_{2}(d, 0)=\inf (, h), 0, T M R(\right.\right.$

$f_{1}:\{0,1,2\} X\{0,1\} \rightarrow\{0,1,2\} X\{0,1\} X\{T M L, T M R\}\left(f_{2}:\{0,1,2\} X\{0,1\} \rightarrow\{0,1,2\} X\{0,1\} X\{T M L, T M R\}\right.$

)$))())))))))) \Rightarrow\left((a(b, c)=d \Leftrightarrow b \operatorname{th}(, c)=d)\left(\exists a_{1}\left(a_{1}=\{a(z, b) \mid(z \in \mathbb{N}(b \in P(\right.\right.\right.$

$b, d D T M[\{0,1,2\},\{0,1\}, g, h, i, j, k](d \in$ poly func $(\forall l(l \in a(\exists m(m \in$ polyfunc (

$\exists n(n \in q(l, m D T M[\{0,1,2\},\{0,1\}, g, h, n, j, k](\forall o(\forall p(p \in \operatorname{poly}$ func $(o, p N D T M[\{0,1,2\},\{0,1\}, g, h, q, j, k]$

$\left.\left.\left.\left.\left.(\forall r(r \in o(r \in a))))))))))))))))))\}\left(\{01\} \in a_{1}\right)\right)\right)\right)\right)\right) \wedge((a(b, c)=d \Leftrightarrow b \operatorname{th}(, c)=d)($

$\exists a_{1}\left(a_{1}=\{a(z, b) \mid(z \in \mathbb{N}(b \in P(b, d D T M[\{0,1,2\},\{0,1\}, g, h, i, j, k](d \in \operatorname{polyfunc}(\forall l(l \in a(\exists m)\right.$
} 
$m \in \operatorname{polyfunc}(\exists n(n \in q(l, m D T M[\{0,1,2\},\{0,1\}, g, h, n, j, k]$

$\left.\left.(\forall o(\forall p(p \in \operatorname{polyfunc}(o, p N D T M[\{0,1,2\},\{0,1\}, g, h, q, j, k](\forall r(r \in o(r \in a)))))))))))))))))))\}\left(\{01\} \in a_{1}\right)\right)\right) \Rightarrow$ $\left(\left(\neg(((a) b, c)=d \Leftrightarrow b \operatorname{th}(, c)=d)\left(a_{1}=\{a(z, b) \mid(z \in \mathbb{N}(b \in P(b \subseteq y(b, d D T M[e, f, g, h, i, j, k](d \in\right.\right.\right.$ polyfunc $\left(\forall l\left(l \in a_{1}(\exists m(m \in\right.\right.$ polyfunc $(\exists n(n \in q(l, m D T M[e, f, g, h, n, j, k](\forall o(\forall p(p \in$ polyfunc ( $\left.\left.\left.\left.\left.\left.\left.\left.\left.\left.\left.\left.\left.\left.\left.\left.\left.\left.\left.o, p N D T M[e, f, g, h, q, j, k]\left(\forall r\left(r \in o\left(r \in a_{1}\right)\right)\right)\right)\right)\right)\right)\right)\right)\right)\right)\right)\right)\right)\right)\right)\right)\right)\right)\right\}\left(\{01\} \in a_{1}\left(a_{1} \in P\right)\right)\right)\right) \Rightarrow$ $\left((a(b, c)=d \Leftrightarrow b \operatorname{th}(, c)=d)\left(\forall a_{1}\left(a_{1}=\{a(z, b) \mid(z \in \mathbb{N}(b \in P(b, d D T M[e, f, g, h, i, j, k](d \in\right.\right.\right.$ polyfunc $(\forall l(l \in a(\exists m(m \in$ polyfunc $(\exists n(n \in q(l, m D T M[e, f, g, h, n, j, k](\forall o(\forall p(p \in$ polyfunc ( $\left.\left.\left.\left.\left.\left.o, p N D T M[e, f, g, h, q, j, k](\forall r(r \in o(r \in a))))))))))))))))))\}\left(\{01\} \in a_{1}\left(a_{1} \notin P\right)\right)\right)\right)\right)\right)\right)\right)$ $\wedge\left(\left(\neg\left((a(b, c)=d \Leftrightarrow b \operatorname{th}(, c)=d)\left(a_{1}=\{a(z, b) \mid(z \in \mathbb{N}(b \in P(b \subseteq y(b, d D T M[e, f, g, h, i, j, k](d \in\right.\right.\right.\right.$ polyfunc $\left(\forall l\left(l \in a_{1}(\exists m(m \in \operatorname{polyfunc}(\exists n(n \in q(l, m D T M[e, f, g, h, n, j, k](\forall o(\forall p(p \in\right.\right.$ polyfunc ( $\left.\left.\left.\left.\left.\left.\left.\left.\left.\left.\left.\left.\left.\left.\left.\left.\left.\left.\left.\left.o, p N D T M[e, f, g, h, q, j, k]\left(\forall r\left(r \in o\left(r \in a_{1}\right)\right)\right)\right)\right)\right)\right)\right)\right)\right)\right)\right)\right)\right)\right)\right)\right)\right)\right)\right\}\left(\{01\} \in a_{1}\left(a_{1} \in P\right)\right)\right)\right)\right) \Rightarrow$ $\left((a(b, c)=d \Leftrightarrow b \operatorname{th}(, c)=d)\left(\forall a_{1}\left(a_{1}=\{a(z, b) \mid(z \in \mathbb{N}(b \in P(b, d D T M[e, f, g, h, i, j, k](d \in\right.\right.\right.$ polyfunc $(\forall l(l \in a(\exists m(m \in$ polyfunc $(\exists n(n \in q(l, m D T M[e, f, g, h, n, j, k](\forall o(\forall p(p \in$ poly func ( $\left.\left.\left.\left.o, p N D T M[e, f, g, h, q, j, k](\forall r(r \in o(r \in a))))))))))))))))))\}\left(\{01\} \in a_{1}\left(a_{1} \notin P\right)\right)\right)\right)\right)\right)$

Th.3. $(\exists a(a \subseteq P(a \notin P(a \in B P P))))$

$\operatorname{Pr} .3(a \subseteq P($ string $[, a]($ string $[, b](|a|=|b|(\forall j(\forall k(\exists l(D T M[c, d, e, f, g, h, i, j \operatorname{th}(, b), k, l]$ $(l=m, n, o((m=\sup (, i) \Leftrightarrow(k \in j \operatorname{th}(, a)))((m=\inf (, i) \Leftrightarrow(k \notin j t h(, a)))(\forall j(N D T M[c, d, e, f, b, h, i, j, k, l]$

$\left.\left(|j| \leqslant \mathbf{R} \mathbf{R}\left(\mathbb{R}_{<, \text {deci }}(10),|k|^{\mathbf{R}_{<, \text {deci }}(100)}\right)\right)\right)(\{k \mid(k \in a \vee(N D T M[c, d, e, f, b, h, i, j, k, l]$

$(l=m, n, o(m=\sup (, i)))))))\}=z(z \notin P)))))))))))))) \Rightarrow(z \in B P P)) \wedge(\exists a(a \subseteq P(\operatorname{string}[, a](\operatorname{string}[, b]$

$(|a|=|b|(\forall j(\forall k(\exists l(D T M[c, d, e, f, g, h, i, j \operatorname{th}(, b), k, l]$

$(l=m, n, o((m=\sup (, i) \Leftrightarrow(k \in j \operatorname{th}(, a)))((m=\inf (, i) \Leftrightarrow(k \notin j \operatorname{th}(, a)))(\forall j(N D T M[c, d, e, f, b, h, i, j, k, l]$

$\left(|j| \leqslant_{\mathbf{R}} \mathbf{R}\left(\mathbb{R}_{<, \text {deci }}(10),|k|^{\left.\left.\left.\mathbf{R}_{<, \text {deci }}(100)\right)\right)\right)(\{k \mid(k \in a \vee(N D T M[c, d, e, f, b, h, i, j, k, l]}\right.\right.$

$(l=m, n, o(m=\sup (, i)))))))\}=z(z \notin P()))))))))))))))$

Theorem.4.

$\mathrm{P}$ is a proper subset of BPP

Proof.4.

There exists a set in BPP that is not in $\mathrm{P}$ and we already have from theorem 1 that $\mathrm{P}$ is a improper subset of BPP

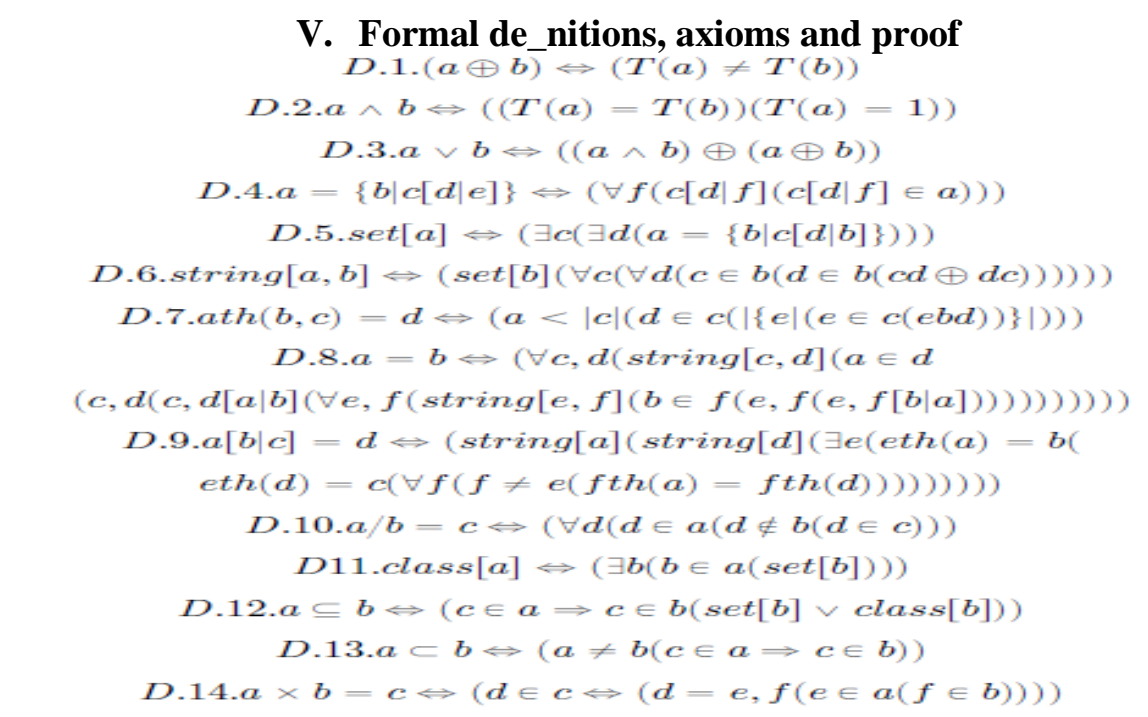

$D .15 . S_{b}^{a}(c)=d \Leftrightarrow(\operatorname{string}[a, b](c \in b(d \in b(\operatorname{cad}(-((\exists f(f a d(\operatorname{caf})))$ 
D.16. $a \simeq^{b, c} d \Leftrightarrow(\forall c, d(\operatorname{string}[c, d](a \in d(c, d((\neg(c, d \Rightarrow(g b h(g \in c(h \in c)))))$

$(c, d[a \mid b](\forall e, f($ string $[e, f]($

$b \in f(e, f((\neg(e, f \Rightarrow(i b j(i \in f(j \in f)))))(e, f[b \mid a]))))))))))))$

D.17. $(a=\{b, c\}(\mid \notin c($ string $[b, c]))) \Leftrightarrow(d \in a \Leftrightarrow((d=\inf (b, c)) \vee(d=\sup (b, c))) \vee(\exists e$

$$
\begin{gathered}
\left.\left.\left(e \simeq^{b, c}=,\left(\exists f\left(f \simeq^{b, c}=,\left(S_{c}^{b}(e)=d\left(S_{c}^{b}(d)=f\right)\right)\right)\right)\right)\right)\right) \\
\text { D.18.(string }[b, c](a \in c(a=b, c))) \Leftrightarrow(c=\{a\})
\end{gathered}
$$

D.19. $(a c b=c, d(\operatorname{string}[c, d])) \Leftrightarrow(b \in d(a=c, e(\operatorname{string}[c, e](e=d /\{b\}(\forall f(f \in e(f c b))))))$

$$
\text { D.20.deci }=\{0,1,2,3,4,5,6,7,8,9\}
$$

D.21. $<$, deci $=0<1<2<3<4<5<6<7<8<9$

$D .22 . \mathbb{N}_{a, b}=\left\{a, c \mid\left(\right.\right.$ string $\left.\left.[a, c]\left(\forall d\left(d \in c\left(d \simeq^{a, c} e(e \in b)\right)\right)\right)\right)\right\} \Leftrightarrow([a, b])$

D.23. $a: b \rightarrow c \Leftrightarrow(\forall d(d \in b(\exists e(e \in c(a(d)=e(\forall f(f \notin b(\forall g(a(f) \neq g)))))))))$

D.24.injection $[a, b, c] \Leftrightarrow(a: b \rightarrow c(a(d)=a(e) \Rightarrow d=e))$

$D .25$.surjection $[a, b, c] \Leftrightarrow(a: b \rightarrow c(\forall d(d \in c(\exists e(e \in b(a(e)=d))))))$

D.26.bijection $[a, b, c] \Leftrightarrow($ injection $[a, b, c](\operatorname{surjection}[a, b, c]))$

$$
\begin{aligned}
& D .27 .|a|<|b| \Leftrightarrow(\exists c(\text { injection }[c, a, b])) \\
& D .28 .|a|=|b| \Leftrightarrow(\exists c(\text { bijection }[c, a, b]))
\end{aligned}
$$

$D .29 . a, b<\mathbb{N}_{a, c} a, d \Leftrightarrow\left(a, b \in \mathbb{N}_{a, c}\left(a, d \in \mathbb{N}_{c, d}(\operatorname{string}[a, b](\operatorname{string}[a, d]((|b|<|d|) \oplus\right.\right.$

$\left.\left.\left.\left.\left.\left(|b|=|d|\left(\exists e\left(\forall f\left(f<e\left(f \operatorname{th}(a, b)=f \operatorname{th}(a, d)\left(\operatorname{eth}(a, b)<\mathbf{N}_{a, c} \operatorname{eth}(a, d)\right)\right)\right)\right)\right)\right)\right)\right)\right)\right)\right)$

$D .30 \mathbb{N}_{a, b}(c)=d \Leftrightarrow\left(c \in \mathbb{N}_{a, b}\left(\left|\left\{e \mid\left(e<\mathbb{N}_{a, b} c\right)\right\}\right|=d\right)\right)$

$D .31 . \mathbb{N}=\left\{a \mid\left(\exists b\left(\exists c\left(\right.\right.\right.\right.$ string $\left.\left.\left.\left.[b]\left(\mathbb{N}_{b}(c)=a\right)\right)\right)\right)\right\}$

$D .32 . T M[a, b, c, d, e, f, g] \Leftrightarrow(c \in b(d=b / c(g \subseteq a(f \in a($

$\left.\left.\left.\left.\left.e:(a / g) \times b \times\{T M L, T M R\} \rightarrow a \times b \times\{T M L, T M R\}\left(|g|=\mathbb{N}_{<, \text {deci }}(2)\right)\right)\right)\right)\right)\right)$

$D .33 . a \lesssim_{b, c} d \Leftrightarrow\left(\left(\operatorname{set}[a] \vee \operatorname{class}[a]\left((\operatorname{set}[d] \vee \operatorname{class}[d])\left(\forall e\left(e \in a\left(e \simeq^{b, c} f(f \in d)\right)\right)\right)\right)\right)\right.$

D.34.compfunc $[a, b] \Leftrightarrow(\operatorname{string}[, a](\forall c(b(c)=d(e(c))(\operatorname{compfunc}[f, e](d=\sup (a)(f=a /\{d\}))))))$

D.35.DTM $[a, b, c, d, e, f, g, h, i, j] \Leftrightarrow(, j=A, B, C(A \in g(B \in b(C \in \mathbb{N}(T M[a, b, c, d, e, f, g]($

string $[, i]$ (string $[, j]\left(i \lesssim b\left(\right.\right.$ compfunc $[h, k]\left(k\left(f,, i, \mathbb{N}_{<, \text {deci }}(0)\right)=, j(|h| \in \mathbb{N}(\right.$

$($ lth $(, h)(m, n, o)=p, q, r) \Leftrightarrow\left(\left(\left(\left(o=\mathbb{N}_{<, \text {deci }}(0)\left(e(m\right.\right.\right.\right.\right.$, inf $(n))=p, \mathbb{N}_{<, \text {deci }}(1), T M L$

$\left.\left.\left(r=\mathbb{N}_{<, \text {deci }}(0)\left(\inf (q)=c\left(\forall s\left(r<_{\mathbb{N}} s\left(\operatorname{sth}(n)=S_{\mathbb{N}}^{<}(s) \operatorname{th}(q)\right)\right)\right)\right)\right)\right)\right) \oplus(\operatorname{oth}(n)=\sup (n)$

$\left.\left.\left(e(m, \sup (n))=p, \operatorname{sth}(q), T M R\left(S_{\mathbb{N}}^{\odot}(0)=r\left(\sup (q)=r \operatorname{th}(q)\left(\operatorname{rth}(q)=c\left(\forall t\left(t<_{\mathbb{N}} o(\operatorname{tth}(n)=\operatorname{tth}(q))\right)\right)\right)\right)\right)\right)\right)\right)$

$\oplus\left(o \neq \mathbb{N}_{<, \operatorname{deci}}(0)(e(m\right.$, oth $\left.\left.(n))=p, \operatorname{oth}(q), \operatorname{TML}(\forall s(s \neq o(\operatorname{sth}(n)=\operatorname{sth}(q)))))\right)\right) \oplus(\operatorname{oth}(n) \neq \sup (n)$

$(e(m, o t h(n))=p, o t h(q), T M R(\forall s(s \neq o(\operatorname{sth}(n)=\operatorname{sth}(q)))))))))))))))))))$

D.36.NDTM $[a, b, c, d, e, f, g, h, i, j] \Leftrightarrow(\forall k(k \in e(T M[a, b, c, d, k, f, g]($

compfunc $[k, l]\left(l\left(f, l, \mathbb{N}_{<, \text {deci }}(0)\right)=, j\right.$

$(, j=A, B, C(A \in g(B \in b(C \in \mathbb{N}(\forall m(m<\mathbb{N}|h|(\exists n(n \in e(m t h(h)(o, p, q)=r, s, t$

$\left(((n), q \operatorname{th}(p))=r, q \operatorname{th}(s), u\left(\left(q \neq \mathbb{N}_{<, \text {deci }}(0)\left(u=T M L\left(S_{\mathbb{N}}^{<}(t)=q\right)\right)\right) \oplus(q \operatorname{th}(p) \neq \sup (p)(u=T M R\right.\right.$

$\left.\left.\left.\left(S_{\mathrm{N}}^{<}(q)=t\right)\right)\right)\right) \oplus\left(n(o, q \operatorname{th}(p))=r, u \operatorname{th}(s), v\left(u=\mathbb{N}_{<, \operatorname{dec} i}(1)\left(\inf (s)=c\left(\forall w\left(w \operatorname{th}(p)=S_{\mathrm{N}}^{<}(w) \operatorname{th}(s)(v=T M L\right.\right.\right.\right.\right.$

$\left.\left.\left.\left.\left.\left.\left(S_{\mathrm{N}}^{<}(t)=q\right)\right)\right)\right)\right)\right)\right) \oplus\left(u=q\left(S_{\mathrm{N}}^{<}(q) \operatorname{th}(s)=\sup (s)(q \operatorname{th}(p)=\sup (p)(v=T M R(\sup (s)=c(\right.\right.$

$\forall w(w<\mathbf{N} q(w t h(p)=w t h(s))))))))))))))))))))))))$

D.37. $\left(a=b^{c}(a \in \mathbb{N})\right) \Leftrightarrow\left(\operatorname{compfunc}[d, e]\left(e\left(\mathbb{N}_{<, \text {deci }}(1)\right)=a(|d|=c(\forall f(f t h(, d)(g)=\right.\right.$ iv $\left.\left.(b, g))))\right)\right)$

D.38.a(b) $=O_{c}(d(b)) \Leftrightarrow\left(\exists e\left(\exists f\left(e \in \mathbb{N}\left(f \in \mathbb{N}\left(\forall g\left(g \in c\left(a(g) \leqslant \mathrm{N}\right.\right.\right.\right.\right.\right.\right.$ N $\left.\left.\left.\left.\left.\left.\left.\left(e, d(g)^{f}\right)\right)\right)\right)\right)\right)\right)\right)$

D.39. $|a| \leqslant|b| \Leftrightarrow((|a|=|b|) \oplus(|a|<|b|))$

D.40. $+\mathbb{N}(a, b)=c \Leftrightarrow(c \in \mathbb{N}(a \in \mathbb{N}(b \in \mathbb{N}(\{d \mid(d<\mathbb{N} c(a \leqslant \mathbb{N} d))\}))))$

$D .41 \cdot \dot{\mathbf{N}}(a, b)=c \Leftrightarrow(c \in \mathbb{N}(a \in h d s N(b \in \mathbb{N}$

$\left.\left.\left.\left(\operatorname{compfunc}[d, e]\left(|d|=b\left(e\left(\mathbb{N}_{<, \text {deci }}(0)\right)=c\left(\forall f\left(\forall g\left(f t h(d)(g)=+_{\mathbb{N}_{<, \text {deci }}}(a, g)\right)\right)\right)\right)\right)\right)\right)\right)\right)$ 
$D .42 \operatorname{invath}(b, c)=d \Leftrightarrow(\mid\{e \mid$ dae $\} \mid=a$

$D .43 . P=\{k \mid((\operatorname{set}[k] \vee \operatorname{class}[k])(\exists a(\exists b(\exists c(\exists d(\exists e(\exists f(\exists g) \exists l(\exists m(\forall i) \exists h(\exists j(N D T M[a, b, c, d, e, f, g, h, i, j]($

$$
|h|=\mathrm{i}\left(l,|i|^{m}(j=n, o, p(n=\inf (, g) \Leftrightarrow\right.
$$

$i \notin k)((n=\sup (, g) \Leftrightarrow i \in k)(n=\sup (, g) \oplus n=\inf (, g))))))))))))))))))))\}$

$D .44 . N P=\{k \mid(\exists a(\exists b)(\exists c(\exists d(\exists e(\exists f(\exists g)(\exists l(\exists m)(\forall i), i \in k(\exists h(\exists j(N D T M[a, b, c, d, e, f, g, h, i, j]$

$$
\left(|h|={ }_{\mathrm{i}}\left(l,|i|^{m}\right)(j=n, o, p(n=\inf (, g) \Leftrightarrow i \notin k)(\right.
$$

$(n=\sup (, g) \Leftrightarrow i \in k)(n=\sup (, g) \oplus n=\inf (, g))))))))))))))))))))\}$

$D .45 . \mathbb{Z}_{a, b}=\left\{a, c \mid\left(\right.\right.$ string $\left.\left.[a, c]\left(a, c \in \mathbb{N}_{a, b} \oplus\left(a, c=-\operatorname{ad}\left(\operatorname{string}[a, d]\left(a, d \in \mathbb{N}_{a, c}\right)\right)\right)\right)\right)\right\}$

$D .46 . \mathbb{Z}_{a}(b)=c \Leftrightarrow\left(\mathbb{N}_{a}(b)=c \oplus\left(b=-\operatorname{de}\left(\operatorname{string}[d, e]\left(c=-\mathbb{N}_{a}(d, e)\right)\right)\right)\right)$

$$
D .47 . \mathbb{Z}=\left\{a \mid\left(\exists b\left(\exists c\left(\mathbb{Z}_{b}(c)=a\right)\right)\right)\right\}
$$

D.48. $\mathbb{R}_{a, b}=\left\{a, c \mid\left(\right.\right.$ string $[a, c]\left(\left(a, c \in\right.\right.$ mathds $Z_{a, b} \oplus\left(. \in c\left(\forall e\left(e \in c\left(\sup (a, c) \neq\left(\forall g\left(g \in c\left(g \simeq^{a, c} h(g \neq .(h \in b\right.\right.\right.\right.\right.\right.\right.$ )$\left.\left.\left.))))))))) \oplus\left(\inf (a, c)=-\left(\sup (a, c) \neq .\left(\forall g\left(g \in c\left(g \neq-\left(g \neq .\left(g \simeq^{a, c} h(h \in h(h \in b))\right)\right)\right)\right)\right)\right)\right)\right)\right)\right\} \Leftrightarrow \operatorname{string}[a, b]$ D.49. $\mathbb{R}_{a}(b)=\mathbb{Z}_{a}(b) \Leftrightarrow b \in \mathbb{Z}_{a}$

$D .50 \cdot \cdot_{\mathbf{Z}_{a, b}}^{+}(a, c, a, d)=a, e \Leftrightarrow\left({\left(\mathbb{Z}_{a, b}^{+}\right.}^{+}(a, c, a, d)=a, e \oplus\left(\dot{\mathbb{Z}}_{a, b}^{+}(a, c /\{\inf (a, c)\}, a, d /\{\inf (a, d)\})=a, e\right.\right.$

$\left.(\inf (a, c)=-(\inf (a, d)=-)))) \oplus\left(\inf (a, d)=-\left(\inf (a, c) \neq-\left(-\mathbf{N}_{a, b}(a, c, a, d /\{\inf (a, d)\})\right)\right)\right)\right)$

$D .51 . \mathbb{R}=\left\{a \mid\left(\exists d\left(\exists b, c\left(\right.\right.\right.\right.$ string $\left.\left.\left.\left.[b, c]\left(\mathbb{R}_{b, c}(d)=a\right)\right)\right)\right)\right\}$

$D .52 \cdot \cdot_{\mathbf{R}_{a, b}}^{+}(c, d)=e \Leftrightarrow\left(\left(\left(\hat{+}_{\mathbb{Z}_{a, b}}(c, d)=e\right) \oplus(((\inf (c)=-(\inf (d)=-)) \oplus(\inf (c) \neq-(\inf (d) \neq-)))\right.\right.$

$\left((\neg(\exists f(f \operatorname{th}(c)=))).\left(g \operatorname{th}(d)=.\left(c=a, i\left(d=a, j{\left(\dot{\mathbf{Z}}_{a, b}^{+}\right.}(c,\{l \mid(l=m \operatorname{th}(d)(m<N g))\})=n(\right.\right.\right.\right.$

$e=a, n \cup\{m \mid(m=\operatorname{oth}(d)(g \leqslant \mathbb{N} o))\}(\sup (a, n) a(a,\{m \mid(m=\operatorname{oth}(d)(g \leqslant \mathbb{N} o))\}))))))))))$

$\oplus(((\inf (c)=-(\inf (d)=-)) \oplus(\inf (c) \neq-(\inf (d) \neq-)))(f \operatorname{th}(c)=.(g \operatorname{th}(d)=.(f \leqslant \mathbb{N} g(\sup (c)=j \operatorname{th}(c)$

$(\sup (d)=k \operatorname{th}(d)(\operatorname{compfunc}[l, m](m(d)=e(n t h(, l)(d)=o \Leftrightarrow((n<\mathbf{N}-\mathbf{N}(k, f)$

$\left(\operatorname{invnth}(o)={\stackrel{+}{\mathbb{N}_{a, b}}}_{+}^{+}(\operatorname{invnth}(c)\right.$, invnth $(d))\left(_{\mathbb{N}_{a, b}}^{+}(\operatorname{invnth}(c)\right.$, invnth $(d))=a, q\left(|q|=\mathbb{N}_{<, \text {deci }}(1)\right.$

$(\forall p(p \neq n(\operatorname{invpth}(o)=\operatorname{invpth}(d)))))) \oplus(n<\mathbf{N}-\mathbb{N}(k, g)(-\mathbb{N}(j, f)<\mathbf{N} n$

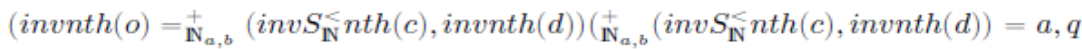

$\left.\left.\left.\left.\left.\left.\left(|q|=\mathbb{N}_{<, \text {deci }}(1)(\forall p(p \neq n(\operatorname{invpth}(o)=\operatorname{invpth}(d))))\right)\right)\right)\right)\right)\right) \oplus(d=o(\operatorname{invnth}(d)=g \operatorname{th}(d)))\right) \oplus$

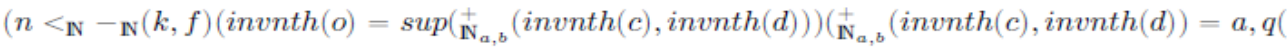

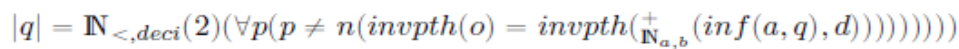

$\oplus\left(n<_{\mathbf{N}}-\mathbf{N}(k, g)\left(-\mathbf{N}(j, f)<\mathbf{N} n\left(\operatorname{invnth}(o)=\sup \left({\stackrel{+}{\mathbb{N}_{a, b}}}^{+}\right.\right.\right.\right.$

$\left(\operatorname{inv} S_{\mathbb{N}}^{<}(n) \operatorname{th}(c)\right.$, invnth $\left.\left.(d)\right)\right)\left(_{\mathbb{N}_{a, b}}^{+}\left(\operatorname{inv} S_{\mathbb{N}}^{<}(n) \operatorname{th}(c), \operatorname{invnth}(d)\right)=a, q\left(|q|=\mathbb{N}_{<, \text {deci }}(2)\right.\right.$

$\left.\left.\left.\left.\left.\left.\left.\left.\left.\left.\left.\left.\left.\left.\left.\left.\left(\forall p\left(p \neq n\left(\operatorname{invpth}(o)=\operatorname{invpth}\left({\left(\mathbb{N}_{a, b}\right.}^{+}(\inf (a, q), d)\right)\right)\right)\right)\right)\right)\right)\right)\right)\right)\right)\right)\right)\right)\right)\right)\right)\right)\right)\right)$

$D .53 .{ }_{\mathbb{R}}^{+}(a, b)=c \Leftrightarrow\left(\exists d, e\left(\operatorname{string}[d, e]\left(\exists f\left(\exists g\left(\exists h\left({\stackrel{\mathbb{R}_{d, e}}{+}}_{f}(f, g)=h\left(\mathbb{R}_{d, e}(f)=a\left(\mathbb{R}_{d, e}(g)=b\left(\mathbb{R}_{d, e}(h)=c\right)\right)\right)\right)\right)\right)\right)\right.\right.$

$$
D .54 \cdot \cdot_{\mathbf{R}}^{+}(a, b)={ }_{\mathbf{R}}^{+}(b, a)
$$

D.55. $\mathbb{Z}_{a, b}(a, c, a, d)=a, e \Leftrightarrow\left(\left(\left(\mathrm{i}_{a, b}(a, c, a, d)=a, e\right)\right.\right.$

$\left.\oplus\left(a, c \in \mathbb{N}_{a, b}\left(\inf (a, d)=-\left(\mathrm{iN}_{a, b}(a, c, a, d /\{\inf (a, d)\})=a, f(a, e=-a a, e)\right)\right)\right)\right)$

$\left.\oplus\left(\inf (a, c)=-\left(\inf (a, d)=-\left(\mathrm{i}_{a, b}(a, c /\{\inf (a, c)\}, a, d /\{\inf (a, d)\})\right)\right)\right)\right)$

$D .56 \cdot \dot{\mathbb{Z}}(a, b)=c \leftrightarrow\left(\exists d\left(\exists e\left(\exists f\left(\exists g\left(\mathbb{Z}_{d}(e, f)=g\left(\mathbb{Z}_{d}(e)=a\left(\mathbb{Z}_{d}(f)=b\left(\mathbb{Z}_{d}(g)\right)\right)\right)\right)\right)\right)\right)\right.$

D.57./ $\mathbb{Z}_{a}(b, c)=d \Leftrightarrow\left(\dot{\mathbf{Z}}_{a}(d, c)=b\right)$

D.58./Z $\mathbb{Z}(a, b)=c \Leftrightarrow(\grave{\mathbf{Z}}(c, b)=a)$

D.59. $\left(\dot{\mathrm{R}} a, b(a, c, a, d)=a, e\left(a, c \in \mathbb{Z}_{a, b} \oplus a, d \in \mathbb{Z}_{a, b}\right)\right) \Leftrightarrow((h(i)=j) \Leftrightarrow(i \in b$

$\left.\left(\operatorname{iasup}(a, b)\left(S_{\mathbf{R}_{a, b}}^{<\mathbf{R}_{a, b}}(\sup (a, b))=k\left(\mathbb{N}_{a, b}(i, j)=k\right)\right)\right)\right)$

$\left(\dot{\mathbf{Z}}_{a, b}(a, c, a,\{l \mid(l=\operatorname{mth}(a, d)(m<g))\})=l(\right.$ compfunc $[m, n]$

$\left.\left.\left.\left.m(l))=a, e\left(\left(q \operatorname{th}(, m)(r)=s \Leftrightarrow s=/ \mathbf{z}_{a, b}\left(r, h\left(+\frac{+}{\mathbb{N}}(q, f) \operatorname{th}(a, d)\right)\right)\right)\right)\right)\right)\right)\right)$

D.60. $\left.\left(\dot{\mathbf{R}}_{a, b}(a, c, a, d)=a, e\left(\neg\left(a, c \in \mathbb{Z}_{a, b} \oplus a, d \in \mathbb{Z}_{a, b}\right)\right)\right)\right) \Leftrightarrow\left(\left(\left(\dot{\mathbf{z}}_{a, b}(a, c, a, d)=a, e\right) \oplus\right.\right.$

$(f \operatorname{th}(a, c)=.(g \operatorname{th}(a, d)=.(((h(i)=j) \Leftrightarrow(i \in b$

$\left.\left(\operatorname{iasup}(a, b)\left(S_{\mathbf{R}_{a, b}}^{<\mathbf{R}_{a, b}}(\sup (a, b))=k\left(\mathbb{N}_{a, b}(i, j)=k\right)\right)\right)\right)$

$\left(\grave{\mathbf{Z}}_{a, b}(a,\{l \mid(l=\operatorname{mth}(a, c)(m<f))\}, a,\{l \mid(l=\operatorname{mth}(a, d)(m<g))\})=l(\operatorname{compfunc}[m, n](\operatorname{compfunc}[o, p]\right.$ 
$\left(o(m(l))=a, e\left(\left(q \operatorname{th}(, m)(r)=s \Leftrightarrow s=/ \mathbf{Z}_{a, b}\left(r, h\left({ }_{\mathbb{N}}^{+}(q, f) \operatorname{th}(a, d)\right)\right)(q \operatorname{th}(, o)(r)=s\right.\right.\right.$ $\left.\left.\left.\left.\left.\left.\left.\left.\left.\left.\left.\Leftrightarrow s=/ \mathbf{Z}_{a, b}\left(r, h\left(\left(_{\mathbb{N}}^{+}(q, g) \operatorname{th}(a, c)\right)\right)\right)\right)\right)\right)\right)\right)\right)\right)\right)\right)\right)\right)$

$D .61 . B P P=\{a \mid(\exists x(\exists y(x \in \mathbb{R}(y \in \mathbb{R}(\exists b, c, d, e, f, g, h,(\forall i(j(i)=\mid\{l \mid(\exists m($

$\left.\left.\left.N D T M[b, c, d, e, f, g, h, l, k, m]\left(|l| \leqslant \mathbf{R} \mathbf{R}\left(x,|k|^{y}\right)\right)\right)\right)\right\} \mid$

$\left(\left((k \in a) \Rightarrow\left(_{\mathbf{R}}\left(j(k), \mathbb{R}_{<, \text {deci }}\left(/_{\mathbf{R}_{, \text {deci }}}(2,3)\right) \leqslant \mathbf{R} \mid\{l \mid(\exists m(N D T M[b, c, d, e, f, g, h, l, k, m]\right.\right.\right.\right.$

$\left.\left.\left.\left.\left.\left.\left(m=n, o, p\left(n=\sup (, h)\left(|l| \leqslant_{\mathbf{R}} \mathbf{R}\left(x,|k|^{y}\right)\right)\right)\right)\right)\right)\right\} \mid\right)\right)\right)\left(\left(k \notin a\left(k \in c^{*}\right)\right) \Rightarrow(\mid\{l \mid(\exists m(N D T M[b, c, d, e, f, g, h, l, k, m]\right.$

$\left.\left.\left.\left.\left.\left.\left.\left.\left.\left.\left.\left.\left.\left.\left(m=n, o, p\left(n=\sup (, h)\left(|l| \leqslant_{\mathbf{R} \mathbf{R}}\left(x,|k|^{l}\right)\right)\right)\right)\right)\right)\right\} \leqslant_{\mathbf{R} \mathbf{R}}\left(\mathbb{R}_{<, \text {deci }}\left(/ \mathbb{R}_{<, \text {deci }}(1,3)\right), j(k)\right)\right)\right)\right)\right)\right)\right)\right)\right)\right)\right)\right\}$

D.62.a, bDTM $[c, d, e, f, g, h, i] \Leftrightarrow(\forall j(\exists k(\exists l(D T M[c, d, e, f, g, h, i, k, j, l](|k| \leqslant b(|j|(l=m, n, o$

$$
((m=\sup (, i) \Leftrightarrow j \in a)
$$$$
(m=\inf (, i) \Leftrightarrow(j \notin a))))))))))
$$

D.63. $\left(a^{b}(b \in \mathbb{N}(a \in \mathbb{N}))\right) \Leftrightarrow\left(\operatorname{compfunc}[c, d]\left(|d|=b\left(\forall e\left(\operatorname{eth}(, c)(f)=\right.\right.\right.\right.$ i $\left.\left.\left.\left.(f, a)\left(d\left(\mathbb{N}_{<, \text {deci }}(1)\right)\right)\right)\right)\right)\right)$

D.64.a polyfunc $\Leftrightarrow\left(\exists d\left(\exists e\left(\forall b\left(a(b)=c \Leftrightarrow\left(c=\right.\right.\right.\right.\right.$ iN $\left.\left.\left.\left.\left.\left(d, b^{e}\right)\right)\right)\right)\right)\right)$

$A x .1 .(\exists a(\operatorname{set}[a]))$

Ax.2. $(\exists a($ class $[a]))$

Ax.4. $\left(\exists a\left(a=\mathbb{N}_{<, \text {deci }}\right)\right)$

Ax.5. $(\exists a(a=\mathbb{N}))$

Th.1.P $\subseteq B P P$

$\operatorname{Pr} .1 .((a \in P) \Rightarrow(\exists b, c, d, e, f, g, h(a, j D T M[b, c, d, e, f, g, h](j \in$ poly func $))))$

$\wedge(((\exists b, c, d, e, f, g, h(a, j D T M[b, c, d, e, f, g, h](j \in$ polyfunc $))) \Rightarrow(\exists x(\exists y(x \in \mathbb{R}(y \in \mathbb{R}(\exists b, c, d, e, f, g, h,(\forall i($ $j(i)=\left|\left\{l \mid\left(\exists m\left(N D T M[b, c, d, e, f, g, h, l, k, m]\left(|l| \leqslant \dot{\mathrm{R}} \mathbf{R}\left(x,|k|^{y}\right)\right)\right)\right)\right\}\right|$

$\left(\left((k \in a) \Rightarrow\left(\dot{R}\left(j(k), \mathbb{R}_{<, \text {deci }}\left(/_{\mathbf{R}_{, \text {deci }}}(2,3)\right) \leqslant \mathbf{R} \mid\{l \mid(\exists m(N D T M[b, c, d, e, f, g, h, l, k, m]\right.\right.\right.\right.$

$\left.\left.\left.\left.\left.\left.\left(m=n, o, p\left(n=\sup (, h)\left(|l| \leqslant_{\mathbb{R}} \mathbf{R}\left(x,|k|^{y}\right)\right)\right)\right)\right)\right)\right\} \mid\right)\right)\right)\left(\left(k \notin a\left(k \in c^{*}\right)\right) \Rightarrow(\mid\{l \mid(\exists m(N D T M[b, c, d, e, f, g, h, l, k, m]\right.$

$\left.\left.\left.\left.\left.\left.\left.\left.\left.\left.\left.\left.\left.\left.\left(m=n, o, p\left(n=\sup (, h)\left(|l| \leqslant_{\mathbf{R} \mathbf{R}}\left(x,|k|^{l}\right)\right)\right)\right)\right)\right)\right\} \leqslant_{\mathbf{R} \mathbf{R}}\left(\mathbb{R}_{<, \text {deci }}\left(/ \mathbb{R}_{<, \text {deci }}(1,3)\right), j(k)\right)\right)\right)\right)\right)\right)\right)\right)\right)\right)\right)\right\}$

Th.2. $(\exists a(a \subseteq P($ string $[, a]($ string $[, b](|a|=|b|(\forall j(\forall k(\exists l(D T M[c, d, e, f, g, h, i, j \operatorname{th}(, b), k, l]$

$(l=m, n, o((m=\sup (, i) \Leftrightarrow(k \in j \operatorname{th}(, a)))((m=\inf (, i) \Leftrightarrow(k \notin j \operatorname{th}(, a)))(\forall j(N D T M[c, d, e, f, b, h, i, j, k, l]$

$\left.\left(|j| \leqslant_{\mathbf{R}} \mathbf{R}\left(\mathbb{R}_{<, \text {deci }}(10),|k|^{\mathbf{R}_{<, \text {deci }}(100)}\right)\right)\right)(\{k \mid(k \in a \vee(N D T M[c, d, e, f, b, h, i, j, k, l]$

$(l=m, n, o(m=\sup (, i)(\exists A(\exists B(B \in \operatorname{polyfunc}(A, B N D T M[c, d, e, f, b, h, i, j, k, l]$

$(\neg(\exists C(C \in \operatorname{polyfunc}(\exists D(A, C D T M[c, d, e, f, \operatorname{Dth}() b, h, i, j, k, l]))))))))))))))),\} \notin P)))))))))))))$

$\operatorname{Pr.2.}\left(\left((((a) b, c)=d \Leftrightarrow b \operatorname{th}(, c)=d)\left(a_{1}=\{a(z, b) \mid(z \in \mathbb{N}(b \in P(b, d D T M[e, f, g, h, y \operatorname{th}(, i), j, k]\right.\right.\right.$

$(d \in$ poly func $(\forall l(l \in a) \exists m(m \in$ polyfunc $(\exists n(n \in q(l, m D T M[e, f, g, h, n, j, k](\forall o(\forall p(p \in$ polyfunc)

$o, p N D T M[e, f, g, h, q, j, k](\forall r(r \in o(r \in a(\exists A(\exists B(B \in \operatorname{poly} f u n c(A, B N D T M[c, d, e, f, b, h, i, j, k, l]$

$(\neg(\exists C(C \in$ polyfunc $(\exists D(A, C D T M[c, d, e, f, D \operatorname{Dth}(, b), h, i, j, k, l])))))))))))))))))))))))))))\}\left(\{01\} \in a_{1}\left(a_{1} \in P\right.\right.$

$$
))) \Rightarrow(a \subset a)) \wedge
$$

$\left(\left(f_{1}(d, 0)=0,0, T M R\left(f_{1}(0,1)=\sup (, h), 1, T M R\left(f_{2}(d, 1)=0,0,, T M R\left(f_{2}(0,0)=\sup (, h), 0, T M R\right.\right.\right.\right.\right.$

$\left(f_{1}(d, 1)=2,0, \operatorname{TMR}\left(f_{1}(2,0)=\inf (, h), 0, T M R\left(f_{1}(2,1)=\inf (, h), 0, T M R\left(f_{2}(0,1)=\inf (, h), 0, T M R(\right.\right.\right.\right.$

$f_{2}(2,0)=\inf (, h), 0, T M R\left(f_{2}(2,1)=,\inf (, h), 0, T M R\left(f_{2}(d, 0)=\inf (, h), 0, T M R(\right.\right.$

$f_{1}:\{0,1,2\} X\{0,1\} \rightarrow\{0,1,2\} X\{0,1\} X\{T M L, T M R\}\left(f_{2}:\{0,1,2\} X\{0,1\} \rightarrow\{0,1,2\} X\{0,1\} X\{T M L, T M R\}\right.$

()$))))))))))) \Rightarrow\left((a(b, c)=d \Leftrightarrow b t h(, c)=d)\left(\exists a_{1}\left(a_{1}=\{a(z, b) \mid(z \in \mathbb{N}(b \in P(\right.\right.\right.$

$b, d D T M[\{0,1,2\},\{0,1\}, g, h, i, j, k]\left(\left(i=f_{1} \oplus i=f_{2}\right)\left(d \in \operatorname{polyfunc}\left(\forall l\left(l \in a_{1}(\exists m(m \in\right.\right.\right.\right.$ polyfunc (

$\exists n(n \in q(l, m D T M[\{0,1,2\},\{0,1\}, g, h, n, j, k](\forall o(\forall p(p \in \operatorname{poly}$ func $(o, p N D T M[\{0,1,2\},\{0,1\}, g, h, q, j, k]$

$\left.\left.\left.\left.\left.\left.\left.\left.\left.\left.\left.\left.\left.\left.\left.\left.\left.\left.\left.\left.\left.\left(\forall r\left(r \in o\left(r \in a_{1}\right)\right)\right)\right)\right)\right)\right)\right)\right)\right)\right)\right)\right)\right)\right)\right)\right)\right)\right\}\left(\{01\} \in a_{1}\right)\right)\right)\right)\right)\right) \wedge((a(b, c)=d \Leftrightarrow b \operatorname{th}(, c)=d)($

$\exists a_{1}\left(a_{1}=\left\{a(z, b) \mid\left(z \in \mathbb{N}\left(b \in P\left(b, d D T M[\{0,1,2\},\{0,1\}, g, h, i, j, k]\left(\left(i=f_{1} \oplus i=f_{2}\right)\left(d \in \operatorname{polyfunc}\left(\forall l\left(l \in a_{1}(\exists m\right.\right.\right.\right.\right.\right.\right.\right.\right.$ $m \in \operatorname{polyfunc}(\exists n(n \in q(l, m D T M[\{0,1,2\},\{0,1\}, g, h, n, j, k]$

$(\forall o(\forall p(p \in \operatorname{poly} \operatorname{func}(o, p N D T M[\{0,1,2\},\{0,1\}, g, h, q, j, k](\forall r(r \in o(r \in a($ 
$\exists A(\exists B(B \in \operatorname{poly} \operatorname{func}(A, B N D T M[c, d, e, f, b, h, i, j, k, l]$

$\left.\left.(\neg(\exists C(C \in \operatorname{polyfunc}(\exists D(A, C D T M[c, d, e, f, \operatorname{Dth}(, b), h, i, j, k, l]))))))))))))))))))))))))))))\}\left(\{01\} \in a_{1}\right)\right)\right) \Rightarrow$ $\left(\left(\neg(((a) b, c)=d \Leftrightarrow b \operatorname{th}(, c)=d)\left(a_{1}=\{a(z, b) \mid(z \in \mathbb{N}(b \in P(b \subseteq y(b, d D T M[e, f, g, h, i, j, k](d \in\right.\right.\right.$ polyfunc

$\left(\forall l\left(l \in a_{1}(\exists m(m \in\right.\right.$ polyfunc $(\exists n(n \in q(l, m D T M[e, f, g, h, n, j, k](\forall o(\forall p(p \in$ polyfunc (

$o, p N D T M[e, f, g, h, q, j, k]\left(\forall r\left(r \in o\left(r \in a_{1}(\exists A(\exists B(B \in \operatorname{poly} f u n c(A, B N D T M[c, d, e, f, b, h, i, j, k, l]\right.\right.\right.$

$(\neg(\exists C(C \in$ polyfunc $(\exists D(A, C D T M[c, d, e, f, D \operatorname{th}(, b), h, i, j, k, l]$

$\left.(2))())())())())())()))())())))())\}\left(\{01\} \in a_{1}\left(a_{1} \in P()\right)\right)\right) \Rightarrow$

$\left((a(b, c)=d \Leftrightarrow b \operatorname{th}(, c)=d)\left(\forall a_{1}\left(a_{1}=\{a(z, b) \mid(z \in \mathbb{N}(b \in P(b, d D T M[e, f, g, h, i, j, k](d \in\right.\right.\right.$ polyfunc

$(\forall l(l \in a(\exists m) m \in$ polyfunc $(\exists n(n \in q(l, m D T M[e, f, g, h, n, j, k](\forall o(\forall p(p \in$ polyfunc

$o, p N D T M[e, f, g, h, q, j, k](\forall r(r \in o(r \in a(\exists A(\exists B(B \in \operatorname{polyfunc}(A, B N D T M[c, d, e, f, b, h, i, j, k, l]$

$(\neg(\exists C(C \in \operatorname{polyfunc}(\exists D(A, C D T M[c, d, e, f, \operatorname{Dth}(, b), h, i, j, k, l])))))))))))))))))))))))))))\}\left(\{01\} \in a_{1}\right.$

$\left.\left.\left.\left.\left.\left(a_{1} \notin P()\right)\right)\right)\right)\right)\right)$

$\wedge\left((\neg((a) b, c)=d \Leftrightarrow b \operatorname{th}(, c)=d)\left(a_{1}=\{a(z, b) \mid(z \in \mathbb{N}(b \in P(b \subseteq y(b, d D T M[e, f, g, h, i, j, k](d \in\right.\right.$ polyfunc

$\left(\forall l\left(l \in a_{1}(\exists m(m \in\right.\right.$ polyfunc $(\exists n) n \in q(l, m D T M[e, f, g, h, n, j, k](\forall o(\forall p(p \in$ polyfunc (

$o, p N D T M[e, f, g, h, q, j, k]\left(\forall r\left(r \in o\left(r \in a_{1}(\exists A(\exists B(B \in \operatorname{poly} f u n c(A, B N D T M[c, d, e, f, b, h, i, j, k, l]\right.\right.\right.$

$(\neg(\exists C(C \in$ polyfunc $(\exists D(A, C D T M[c, d, e, f, D \operatorname{th}(, b), h, i, j, k, l]$

$\left.\left.(2))())())())())())())()))()))())\}\left(\{01\} \in a_{1}\left(a_{1} \in P()\right)\right)\right)\right) \Rightarrow$

$\left((a(b, c)=d \Leftrightarrow b \operatorname{th}(, c)=d)\left(\forall a_{1}\left(a_{1}=\{a(z, b) \mid(z \in \mathbb{N}(b \in P(b, d D T M[e, f, g, h, i, j, k](d \in\right.\right.\right.$ polyfunc

$(\forall l(l \in a(\exists m) m \in$ polyfunc $(\exists n(n \in q(l, m D T M[e, f, g, h, n, j, k](\forall o(\forall p(p \in$ polyfunc)

$o, p N D T M[e, f, g, h, q, j, k](\forall r(r \in o(r \in a(\exists A(\exists B(B \in \operatorname{polyfunc}(A, B N D T M[c, d, e, f, b, h, i, j, k, l]$

$(\neg(\exists C(C \in$ polyfunc $(\exists D(A, C D T M[c, d, e, f, D$ th $(, b), h, i, j, k, l]$

()$\left.\left.\left.))())()))()))()))()))))())))\}\left(\{01\} \in a_{1}\left(a_{1} \notin P()\right)\right)\right)\right)\right)$

Th.3. $(\exists a(a \subseteq P(a \notin P(a \in B P P))))$

$\operatorname{Pr} .3(a \subseteq P($ string $[, a]$ (string $[, b](|a|=|b|(\forall j(\forall k(\exists l(D T M[c, d, e, f, g, h, i, j \operatorname{th}(, b), k, l]$

$(l=m, n, o((m=\sup (, i) \Leftrightarrow(k \in j \operatorname{th}(, a)))((m=\inf (, i) \Leftrightarrow(k \notin j \operatorname{th}(, a)))(\forall j(N D T M[c, d, e, f, b, h, i, j, k, l]$

$\left.\left(|j| \leqslant_{\mathbf{R} R}\left(\mathbb{R}_{<, \text {deci }}(10),|k|^{\mathbb{R}_{<, \text {deci }}(100)}\right)\right)\right)(\{k \mid(k \in a \vee(N D T M[c, d, e, f, b, h, i, j, k, l]$

$(l=m, n, o(m=\sup (, i)(\exists A(\exists B(B \in \operatorname{polyfunc}(A, B N D T M[c, d, e, f, b, h, i, j, k, l](\neg(\exists C(C \in \operatorname{polyfunc}(\exists D)$

$A, C D T M[c, d, e, f, \operatorname{Dth}(, b), h, i, j, k, l])))))))))))))))\}=z(z \notin P()))))))))))))) \Rightarrow(z \in B P P)) \wedge$

$(\exists a(a \subseteq P($ string $[, a]$ (string $[, b]$

$(|a|=|b|(\forall j(\forall k)(\exists l(D T M[c, d, e, f, g, h, i, j \operatorname{th}(, b), k, l]$

$(l=m, n, o((m=\sup (, i) \Leftrightarrow(k \in j \operatorname{th}(, a)))((m=\inf (, i) \Leftrightarrow(k \notin j \operatorname{th}(, a)))(\forall j(N D T M[c, d, e, f, b, h, i, j, k, l]$

$\left.\left(|j| \leqslant_{\mathbf{R} R}\left(\mathbb{R}_{<, \text {deci }}(10),|k|^{\mathbf{R}_{<, d e c i}(100)}\right)\right)\right)(\{k \mid(k \in a \vee(N D T M[c, d, e, f, b, h, i, j, k, l]$

$(l=m, n, o(m=\sup (, i)(\exists A(\exists B(B \in \operatorname{polyfunc}(A, B N D T M[c, d, e, f, b, h, i, j, k, l]$

$(\neg(\exists C(C \in \operatorname{poly} \operatorname{func}(\exists D($

$A, C D T M[c, d, e, f, \operatorname{Dth}(, b), h, i, j, k, l])))))))))))))))\}=z(z \notin P()))))))))))))))$

Th.4.P $\subset B P P$

$\operatorname{Pr.4.}((\exists a(a \notin P(a \in B P P))) \wedge(P \subseteq B P P))$

VI. English nomenclature

$\mathrm{a} \mathrm{L} b$ in this section means every formal mathematical statement in the languageof the paper about $\mathrm{b}$ has an equivalent formation in the english language about

a

N.1

$\mathrm{a}$ is an subset of $\mathrm{b} \Leftrightarrow a \subseteq b$

$N .2$

$\mathrm{a}$ iff $\mathrm{b} \Leftrightarrow(a \Leftrightarrow b)$

$N .3$

a is a set $\Leftrightarrow \operatorname{set}[a]$

N.4.

a is a state set for some deterministic configuration that solves b 


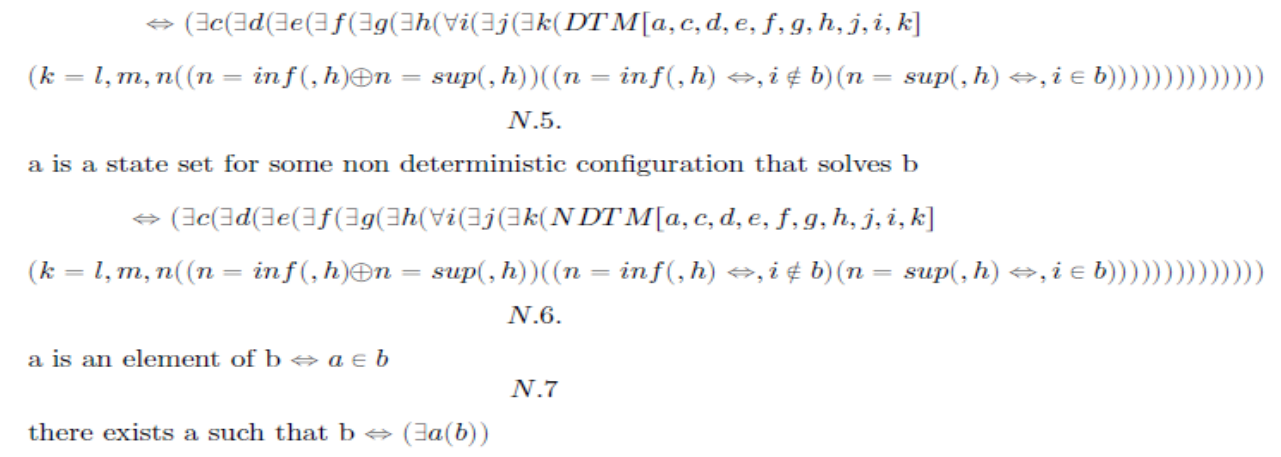

N.8.

for every a such that $\mathrm{b} \mathrm{c} \Leftrightarrow(\forall a(b(c)))$

N.9.

if a then $\mathrm{b} \Leftrightarrow a \Rightarrow b$

N.10.

It is not true that $\mathrm{a} \Leftrightarrow(\neg(a))$

$\mathrm{a}$ is not an element of $\mathrm{b} \Leftrightarrow a \notin b$

N.12.

$\mathrm{a}$ is a superset of $\mathrm{b} \Leftrightarrow b \subseteq a$

cardinality of a L $|a|$

N.13.

N.14.

delta 2 of a that solves b L $\delta_{b}^{2}(a)$

N.15.

$\mathrm{a}$ is a proper subset of $\mathrm{b} \Leftrightarrow a \subset b$

$N .16$

$\mathrm{a}$ is an improper subset of $\mathrm{b} \Leftrightarrow a \subseteq b$

\section{Bibliography}

[1]. Navarro, Gonzalo "A guided tour to approximate string matching".p.7 (2001)ACM Computing Surveys

[2]. Stephen Cook" http://www.claymath.org/sites/default/_les/pvsnp.pdf" (2000)Clay mathematics institute 3.Rogers, H. "Theory of Recursive Functions and Ef-fective Computability." Cambridge, MA: MIT Press, 1987.

[3]. Rogers, H. "Theory of Recursive Functions and E_ective Computability." Cam-bridge, MA: MIT Press, 1987.

[4]. HenkBarendregt, Wil Dekkers, Richard Statman. "Lambda Calculus withTypes", Association of Symbolic Logic (2013)

[5]. HaoWang . "A variant to Turing's theory of computing machines",(1957)Journal of the Association for Computing Machinery (JACM) pp.63\{92.

[6]. Stephen Kleene "Introduction to Metamathematics",Walters-Noordho_, North-Holland, (1952)

[7]. T. P. Baker, J. Gill, R. Solovay. "Relativizations of the P =? NP Question",SIAM Journal on Computing, pp431 \{442 (1975)

[8]. AA Razborov "www.cs.cmu.edu/rudich/papers/natural.ps" (1996)

[9]. S. Aaronson and A. Wigderson (2008)"Algebrization: A New Barrier inComplexity Theory" Proceedings of ACM STOC'2008.(2008) pp. 731\{740.

[10]. Turing, A.M. (1936). "On Computable Numbers, with an Application to theEntscheidungs problem" Proceedings of the London Mathematical Society. 2(1937) Reprint pp115 - 152. 12.Turing, A.M. (1938). "On Computable Numbers, with an Application to the Entscheidungsproblem: A correction'. Proceedingsof the London Mathematical Society. 2 (1937) Reprint pp152-154 Reprinted inMartin Davis.ed. The Undecidable Dover(2004)

[11]. 13.Adrian-Horia, Lozano, Manuel, Mart_n-Vide, Carlos (Eds.) "Theory andPractice of Natural Computing" Springer Verlag (2014)

[12]. Lassaigne, Richard, Rougemont, Michel de "Logic and Complexity" SpringerVerlag (2004)

[13]. Prof. Dr. Lane A. Hemaspaandra, Prof. Dr. MitsunoriOgihara "TheComplexity Theory Companion" Springer Verlag(2002)

[14]. Stephen Cook, Phuong Nguyen "Logical Foundations of Proof Complexity"Association of Symbolic Logic(2010)

[15]. Boaz Barak, Sanjeev Arora "Computational Complexity A Modern Approach"Cambrige University Press. (2009)

[16]. ed. Mikhail J. Atallah, Marina Blanton "Algorithms And Theory Of Com-putation Handbook vol.1. General Concepts And Techniques" CRC. (2009)

[17]. ed. Mikhail J. Atallah, Marina Blanton "Algorithms And Theory Of Com-putation Handbook vol.2. Special Topics And Techniques' CRC. (2009)

[18]. Eric. W.Weinsstein "CRC Encyclopedia Of Mathematics" CRC (2009)

[19]. Flum, J., Grohe, M. "Parametrized Complexity Theory" Springer Verlag(2006)

[20]. ed.Hemaspaandra, Lane, Selman, Alan L. "Complexity Theory Retrospective2" Springer Verlag (1997)

[21]. Elaine Rich "Automata, Computability and Complexity: Theory and Appli-cations " Prentice Hall (2007)

[22]. Steven Homer, Alan L. Selman "Computability And Complexity Theory"Springer Verlag (2011)

[23]. Rodney G. Downey, Denis R. Hirschfeldt "Algorithmic Randomness andComplexity (Theory and Applications of Computability)" Springer Verlag (2010) 
[24]. Alexander Razborov, Steven Rudich "Natural Proofs, Journal of Computerand System Sciences, Vol. 55, No. 1, 1997, pp. 24-35." William I. Gasarch."The Second P=?NP poll". SIGACT News 74.

[25.] Rodney G. Downey, Denis R. Hirschfeldt "Algorithmic Randomness and Complexity (Theory and Applications of Computability)" Springer Verlag (2010) \I

[26.]AlexanderRazborov, Steven Rudich "Natural Proofs, Journal of Computer and System Sciences, Vol. 55, No. 1, 1997, pp. 24-35."

[27.] William I. Gasarch. "The Second P=?NP poll". SIGACT News 74.

[28.]https://complexityzoo.uwaterloo.ca/Complexity_Zoo:B\#bpp 